Rev. Inst. Flor. v. 27 n. 1 p. $73-812015$

http://dx.doi.org/10.4322/rif.2015.006

ISSN impresso 0103-2674/on-line 2178-5031

\title{
CARACTERIZAÇÃo QUíMICA, FÍSICA E MECÂNICA DE BRIQUETES DE DUAS VARIEDADES DE BAMBU ${ }^{1}$
}

\section{CHEMICAL, PHYSICAL AND MECHANICAL CHARACTERIZATION OF BRIQUETTES FROM TWO BAMBOO VARIETIES}

\author{
Paula Martucheli AMARAL 2,3; Fábio Minoru YAMAJI²; \\ Pamela Beatriz Moreira de OLIVEIRA'; \\ Diego Aleixo da SILVA²; José Mauro Santana da SILVA²; Saulo Philipe Sebastião GUERRA²
}

\begin{abstract}
RESUMO - A demanda por energia de fontes renováveis vêm aumentando a cada dia, propiciando o uso de biomassa para que esta possa vir a ser uma alternativa viável como combustível sólido, sendo esta limpa e potencialmente expressiva em termos econômicos. Investigações sobre briquetes para uso doméstico também têm aumentado, pois este material compacto é de fácil transporte e apresenta elevado poder energético, além de contribuir para a preservação ambiental ao substituir matérias-primas não renováveis. O objetivo do presente trabalho foi caracterizar física, mecânica e quimicamente os briquetes de resíduos oriundos de Bambusa vulgaris var. vitatta e Bambusa vulgaris var. vulgaris, analisando o seu potencial para a produção de combustível sólido. Os teores de cinzas e voláteis reportados para a variedade vitatta foram de 7,19 e 75,54\% na devida ordem, e para a variedade vulgaris, de 7,09 e 73,95\%, respectivamente. O produto final de cada material compactado teve expansão longitudinal considerada normal, sendo estas de 7,3\% para vitatta e 7,5\% para vulgaris e apresentaram boa resistência à compressão suportando forças de 510,44 e 499,80 N, respectivamente, para variedade vitatta e vulgaris. Portanto, os briquetes produzidos usando-se resíduos das duas espécies estudadas neste trabalho, Bambusa vulgaris var. vitatta e Bambusa vulgaris var. vulgaris, apresentaram propriedades químicas, físicas e mecânicas satisfatórias para seu uso final como combustível sólido.
\end{abstract}

Palavras-chave: biomassa; Bambusa vulgaris var. vitatta; Bambusa vulgaris var. vulgaris; bioenergia.

\begin{abstract}
Nowadays, the request for renewable energy sources are increasing, leading to the use of biomass as a viable alternative for solid fuel, being cleaner and potentially significant regarding to economical values. The search for domestic use of briquettes has also increased because the solid compact has high source of energy power and can be easily transported, as well as contributes to environmental preservation as a substitute to the use of non-renewable materials. The objective of this study was the physical, mechanical and chemical characterization of briquettes produced with waste of two bamboo varieties, Bambusa vulgaris var. vitatta and Bambusa vulgaris var. vulgaris, analyzing their potential for solid fuel production. The ash and volatile contents reported for vitatta variety were 7.19 and $75.54 \%$ in proper order, and for vulgaris variety were of 7.09 and $73.95 \%$ respectively.
\end{abstract}

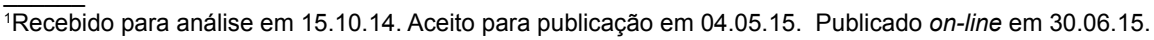

2Universidade Federal de São Carlos - UFSCar - campus Sorocaba, Rodovia João Leme dos Santos (SP-264), Km 110, Bairro do Itinga, 18052-780 Sorocaba, SP, Brasil.

${ }^{3}$ Autor para correspondência: Paula Martucheli Amaral - paulaa.martucheli@gmail.com
} 
The final product of each compacted material had a longitudinal expansion considered normal, being that of $7.4 \%$ for vitatta and $7.5 \%$ for vulgaris, and presented a proper compressive strength, tolerating forces of 510.44 and $499.80 \mathrm{~N}$, for vitatta and vulgaris varieties respectively. Therefore, the briquettes produced in this study using the waste of the two species Bambusa vulgaris var. vitatta and Bambusa vulgaris var. vulgaris, have presented satisfactory chemical, physical and mechanical properties for the final use as solid fuel.

Keywords: biomass; briquettes; Bambusa vulgaris var. vitatta; Bambusa vulgaris var. vulgaris; bioenergy.

\section{INTRODUÇÃO}

Novas pesquisas científicas relacionadas à potencialidade de implementação das energias alternativas estão sendo desenvolvidas em virtude de estas serem de origem renovável e por possuírem baixo potencial poluidor do meio ambiente (Protásio et al., 2014).

A biomassa de proveniência florestal permite diversos usos como fonte alternativa de energia, seja pela queima da madeira, sua conversão em carvão, aproveitamento de resíduos da exploração e aproveitamento de óleos essenciais, alcatrão e ácido pirolenhoso (Cortez et al., 2009).

A compactação de resíduos de biomassa, técnica utilizada para produzir pellets e briquetes, consiste na aglomeração de partículas finas por meio de pressão, com auxílio ou não de um ou mais aglutinantes, resultando em um sólido compacto de volume reduzido com maior eficiência energética, que pode ser armazenado e transportado de forma mais econômica (Carvalho e Brinck, 2004).

Nesse contexto, entender as propriedades da biomassa é essencial para o conhecimento e adequada aplicabilidade de diferentes matérias-primas. Entre elas, pode-se citar densidade, teor de umidade, granulometria, teor de cinzas e de voláteis, carbono fixo, expansão longitudinal, resistência à compressão. Cada propriedade estudada torna-se um ponto relevante para a destinação final do material estudado como combustível (Quirino, 1991).

Particularmente, para o Brasil, país que utiliza intensamente biomassa para atendimento de suas necessidades energéticas, o bambu poderá ser uma importante alternativa às espécies florestais (Brito et al., 1987). A abundância dessa espécie, o rápido crescimento e seu ciclo de corte curto (5 anos), a tornam um recurso alternativo na produção energética (Liu, 2014).

Segundo Ciaramello (1968), as espécies de bambus de maior ocorrência no Brasil são: Phyllostachys sp., Bambusa var. vitatta A. \& C. Rivieri, Dendrocalamus giganteus Munro, Bambusa vulgaris var. vulgaris Schrad ex. Wendl e Bambusa tuldoides Munro.

B. vulgaris var. vitatta é uma espécie popularmente conhecida como bambu-imperial, bambu-verde-amarelo ou bambu-listrado (Cândido e Ribeiro, 1991; Cerri, 1991). Seus colmos apresentam coloração amarela com listras verdes e podem atingir até $17 \mathrm{~m}$ de altura e $16 \mathrm{~cm}$ de diâmetro, a $1 \mathrm{~m}$ do solo. Suas fibras possuem excelentes características, sendo uma delas a elevada resistência mecânica (Albertini, 1979).

Conhecido comumente como bambu-verde, bambu-verde-grande e bambu-comum, a espécie B. vulgaris var. vulgaris foi introduzida pelos portugueses. Essa espécie apresenta colmos de coloração verde, tendendo a amarelo quando se encontram em estágio de envelhecimento, ou quando expostos à incidência direta do sol, e pode atingir até de $15 \mathrm{~m}$ de altura e de 5 a $10 \mathrm{~cm}$ de diâmetro (Albertini, 1979; Cândido e Ribeiro 1991; Cerri, 1991).

Este trabalho objetivou a caracterização e compactação de resíduos das espécies de bambu, Bambusa vulgaris var. vitatta e Bambusa vulgaris var. vulgaris, e a análise do potencial destas matérias-primas como biocombustível sólido. 


\section{MATERIAL E MÉTODOS}

\subsection{Caracterização dos Materiais do Estudo}

Para a realização do experimento foram utilizadas duas variedades de bambu, Bambusa vulgaris var. vitatta e a Bambusa vulgaris var. vulgaris de idade e procedência não determinadas.

Os materiais do estudo foram compostos por todas as partes dos colmos dos bambus produzidos e colhidos pelo produtor. Após sua coleta, foram armazenados em sacos plásticos e selados, para não adquirir umidade ambiente.

\subsection{Densidade a Granel}

Para obter-se a densidade a granel dos materiais, utilizou-se da adaptação da metodologia prescrita na NBR 6922 (Associação Brasileira de Normas Técnicas - ABNT, 1981), assim como Chrisostomo (2011).

\subsection{Classificação e Tamanho das Partículas}

A classificação do tamanho das partículas de cada material foi determinada pelo ensaio de granulometria a granel, baseado na NBR 7217 (Associação Brasileira de Normas Técnicas - ABNT, 1987). O ensaio foi realizado com o peneiramento mecânico, utilizando-se quatro peneiras com abertura de malha progressiva, nas aberturas de 20 mesh $(0,85 \mathrm{~mm}), 35$ mesh $(0,50 \mathrm{~mm}), 60$ mesh $(0,25 \mathrm{~mm}), 100$ mesh $(0,15 \mathrm{~mm})$ e o fundo $(<0,15 \mathrm{~mm})$. O conjunto de peneiras foi instalado em um agitador orbital, marca MARCONI, modelo MA 750, com batidas intermitentes no topo e sistema de agitação mecânica orbital e constante de $250 \mathrm{rpm}$. Foi empregado $100 \mathrm{~g}$ de amostra e a agitação mecânica ocorreu por 10 minutos. A porcentagem de amostra retida nas peneiras é expressa pela Equação 1.

$$
\% \mathrm{MR}=\frac{\mathrm{mR}}{\mathrm{mT}} 100
$$

Em que: $\% M$ representa o material retido em cada peneira, em \%; $m R$ é a massa retida acumulada na peneira, em g, e $m T$ é a massa total da amostra, em g.

\subsection{Determinação dos Teores de Umidade}

Adotou-se como referencial a norma NBR 8112 (Associação Brasileira de Normas Técnicas - ABNT, 1986), secando-se, o material, em estufa a $(105 \pm 2){ }^{\circ} \mathrm{C}$, com o posterior resfriamento em dessecador com sílica gel e com auxílio de uma balança determinadora de umidade, da marca A\&D Company modelo MX - 50 (capacidade $51 \mathrm{~g}$ x legibilidade $0,001 \mathrm{~g}$ ).

\subsection{Determinação do Teor de Voláteis}

Para a determinação do teor de voláteis, os experimentos foram realizados com base na norma NBR 8112 da ABNT, assim como o proposto por Gonçalves et al. (2013). Utilizou-se para as amostras do experimento os materiais moídos em um micromoinho de facas tipo Wiley, seguidos de secagem em estufa $(105 \pm 2)^{\circ} \mathrm{C}$.

\subsection{Determinação do Teor de Cinzas}

A determinação do teor de cinzas foi realizada segundo a ABNT - NBR 8112, com amostras pós-moagem e secagem em estufa $(105 \pm 2){ }^{\circ} \mathrm{C}$, tal como Gonçalves et al. (2013).

\subsection{Determinação do Teor de Carbono Fixo}

O cálculo do teor de carbono fixo foi realizado da mesma maneira que Gonçalves et al. (2013) em seu estudo.

\subsection{Preparação dos Biocombustíveis Sólidos}

Foram confeccionados briquetes homogêneos de cada material, utilizando-se para cada amostra $20 \mathrm{~g}$ do material de origem com um teor de umidade de $10,10 \%$. Para a compactação dos resíduos utilizou-se um molde cilíndrico de aço inoxidável, com diâmetro de $35 \mathrm{~mm}$, em uma prensa hidráulica com capacidade de $30 \mathrm{tf}$, modelo MA/098CP. O processo de compactação foi feito sob pressão de aproximadamente $122 \mathrm{MPa}$, por um tempo de $30 \mathrm{~s}$, sem aquecimento ou uso de aglutinantes. 


\subsection{Aferição da Expansão}

Para analisar a expansão longitudinal, foi realizada a medição da altura (direção de compactação) dos briquetes com um paquímetro digital da marca INSIZE, modelo 1106-502, com capacidade de $150 \mathrm{~mm}$ e precisão de $0,01 \mathrm{~mm}$, nos intervalos de $0,1,2,4,6,12,18,24,48,72$ e 96 horas. Os combustíveis sólidos foram mantidos em uma umidade de equilíbrio a $12 \%$. A expansão longitudinal foi calculada com a Equação 2.

$$
\mathrm{E}=\frac{1 \mathrm{~F}-10}{10} * 100
$$

Em que: $E=$ expansão percentual, em $\% ; l_{F}=$ comprimento da amostra após determinado tempo de compactação em $\mathrm{mm} ; l_{0}=$ comprimento inicial da amostra (logo após compactação), em mm.

Também foi verificada a expansão diametral utilizando-se a Equação 3.

$$
E=\frac{c \mathrm{~F}-c \mathbf{0}}{c 0} * 100
$$

Em que: $E=$ expansão percentual, em $\% ; c F=$ diâmetro da amostra após determinado tempo de compactação em mm; $c 0$ = diâmetro inicial da amostra (logo após compactação), em mm.

\subsection{Ensaio Mecânico}

O comportamento mecânico dos briquetes foi avaliado por meio do teste de tração por compressão diametral, adaptado da norma NBR 7222 (Associação Brasileira de Normas Técnicas ABNT, 2011) para concretos. A máquina utilizada para a realização de ensaios é universal, com capacidade máxima de $30 \mathrm{tf}(300 \mathrm{kN})$, marca EMIC, modelo DL 30.000. Utilizou-se 12 corpos-de-prova de cada material. A velocidade da aplicação da carga utilizada para esse ensaio foi de $3 \mathrm{~mm} \cdot \mathrm{min}^{-1}$.

\section{RESULTADOS E DISCUSSÃO}

\subsection{Teor de Umidade e Cálculo da Densidade a Granel}

Os teores obtidos para umidade e a densidade a granel de cada espécie, após sua chegada ao laboratório, estão apresentados na Tabela 1.

$\mathrm{O}$ valor encontrado neste trabalho para a variedade vitatta se aproxima ao valor reportado por Bianchini et al. (2012) para um diferente material, o bagaço de cana-de-açúcar, de densidade $209,71 \mathrm{~kg} \cdot \mathrm{m}^{-3}$. Já o material da variedade vulgaris apresentou uma densidade a granel próxima a resíduos de vegetais amazônicos encontrados por Pinheiro et al. (2005), com densidade de aproximadamente $400 \mathrm{kgm} . \mathrm{m}^{-3}$ para casca de amêndoa e $500 \mathrm{~kg} . \mathrm{m}^{-3}$ para mistura de maçaranduba com casca de amêndoa.

Tabela 1. Teor de umidade e densidade a granel dos materiais estudados.

Table 1 . Humidity content and bulk density of the studied materials.

\begin{tabular}{ccc}
\hline Espécie & Teor de umidade (\%) & Densidade a granel $\left(\mathrm{kg} \cdot \mathrm{m}^{3}\right)$ \\
\hline Variedade vitatta & 13 & 130 \\
Variedade vulgaris & 10 & 460 \\
\hline
\end{tabular}


A diferença entre os valores encontrados para densidade a granel dos materiais estudados pode ser explicada pela quantidade de fibras e feixes vasculares, os quais são tecidos anatômicos presentes nos colmos de bambu e que variam para cada espécie, influenciando diretamente na densidade do material (Azzini et al., 1977). Outras circunstâncias que explicam essa diferença são as condições de sítio dos materiais coletados, assim como a idades das touceiras e colmos e o material genético das espécies (Brito et al., 1987).
Para o emprego de materiais na produção de combustíveis sólidos, maiores valores de densidade são altamente favoráveis, uma vez que quanto maior sua densidade, maior será a massa contida de energia estocada em um menor volume, resultando assim em compactos mais densos.

\subsection{Granulometria}

$\mathrm{Na}$ Tabela 2, apresentam-se os valores obtidos no ensaio realizado quanto ao tamanho de partículas.

Tabela 2. Distribuição dos tamanhos das partículas das espécies Bambusa vulgaris var. vitatta e Bambusa vulgaris var. vulgaris.

Table 2. Size distribution of Bambusa vulgaris var. vitatta and Bambusa vulgaris var. vulgaris.

\begin{tabular}{cccc}
\hline & Peneiras & \multicolumn{2}{c}{ \% Retida por peneira } \\
\hline Tyler (mesh) & Abertura $(\mathrm{mm})$ & Variedade vitatta & Variedade vulgaris \\
\hline 20 & 0,85 & 10,96 & 0,45 \\
35 & 0,5 & 51,99 & 30,14 \\
60 & 0,25 & 18,57 & 36,63 \\
100 & 0,15 & 9,77 & 18,07 \\
Fundo & $<0,15$ & 8,98 & 14,24 \\
\hline
\end{tabular}

Para os dois materiais estudados, notou-se uma desuniformidade no tamanho das partículas, assim, realizou-se a posterior moagem das matérias-primas buscando-se maior homogeneidade.

Sabe-se que a análise da granulometria das partículas utilizadas na produção dos briquetes é de grande valia, pois o tamanho das partículas pode influenciar diretamente na densidade da biomassa e, consequentemente, na densidade dos briquetes (Protásio et al., 2011).
Busca-se, também, maior homegeneidade das partículas para um melhor empacotamento das mesmas quando compactadas, o que pode influenciar posteriormente na expansão do sólido, afetando seu posterior transporte e armazenamento.

\subsection{Teor de Voláteis, Cinzas e Carbono Fixo}

Os teores de voláteis, cinzas e carbono fixo reportados neste estudo estão apresentados na Tabela 3.

Tabela 3. Resultados da análise dos teores de voláteis, cinzas e cálculo do carbono fixo para os materiais estudados.

Table 3. Ash, volatile and fixed carbon content of the studied materials.

\begin{tabular}{cccc}
\hline Espécie & Teor de voláteis (\%) & Teor de cinzas (\%) & Carbono fixo (\%) \\
\hline Variedade vitatta & 75,54 & 07,19 & 17,27 \\
Variedade vulgaris & 73,95 & 07,09 & 18,96 \\
\hline
\end{tabular}


Costa (2004) encontrou, para o teor de materiais voláteis, o valor de $75 \%$ para Bambusa vulgaris, o qual se aproxima aos relatados neste trabalho para os dois materiais estudados, que são provenientes da mesma espécie. Esse teor está diretamente ligado aos compostos orgânicos presentes no material e que apresentam alto e/ou baixo poder calorífico, influenciando, assim, seu poder de combustão e queima (Barros, 2014).

Em experimento realizado por Gomide et al. (1981), o valor do teor de cinzas relatado para B. vulgaris estava entre 2,2 e $3,2 \%$, enquanto o valor obtido por Junior et al. (2011) foi de 2,06\% para B. vulgaris Schrad. Portanto, os teores de cinzas para os dois materiais analisados neste trabalho podem ser considerados altos se comparados com os valores reportados na literatura. Um dos fatores que influencia nesse teor em espécies de bambu é a composição química dos colmos, que podem apresentar alta taxa de sílica (Brito et al., 1987). Além desse fator, outros, como composição anatômica dos materiais lignocelulósicos, idade, espécie, variedade do material e local de extração das fibras, são exemplos de características ligadas a essa propriedade (Junior et al., 2011).
Esse teor interfere diretamente na determinação do uso final dos briquetes formados por essa matéria-prima, tornando-se, então, uma das principais propriedades a ser analisada na biomassa para fins energéticos (Flores, 2010).

Em relação aos teores de carbono fixo calculados neste trabalho, estes são compatíveis aos reportados por Kleinlein (2010) de 18,47, 16,97 e $19,09 \%$ para as variedades vulgaris, vitatta e D. giganteus, respectivamente. Esse parâmetro é relevante na função da aplicação do produto, uma vez que maiores teores de carbono fixo remetem a carvões de melhor qualidade (Brito et al., 1987).

\subsection{Expansão Longitudinal e Variação Diametral}

Os materiais das duas variedades se mostraram eficazes na produção de briquetes, com a formação de compactos sem desprendimento do material, além de não apresentarem diferenças em relação ao formato, uma vez que o mesmo molde cilíndrico de metal foi utilizado na compactação dos materiais (Figura 1).

O resultados das expansões longitudinal e diametral dos materiais aqui estudados foram apresentados na Tabela 4.
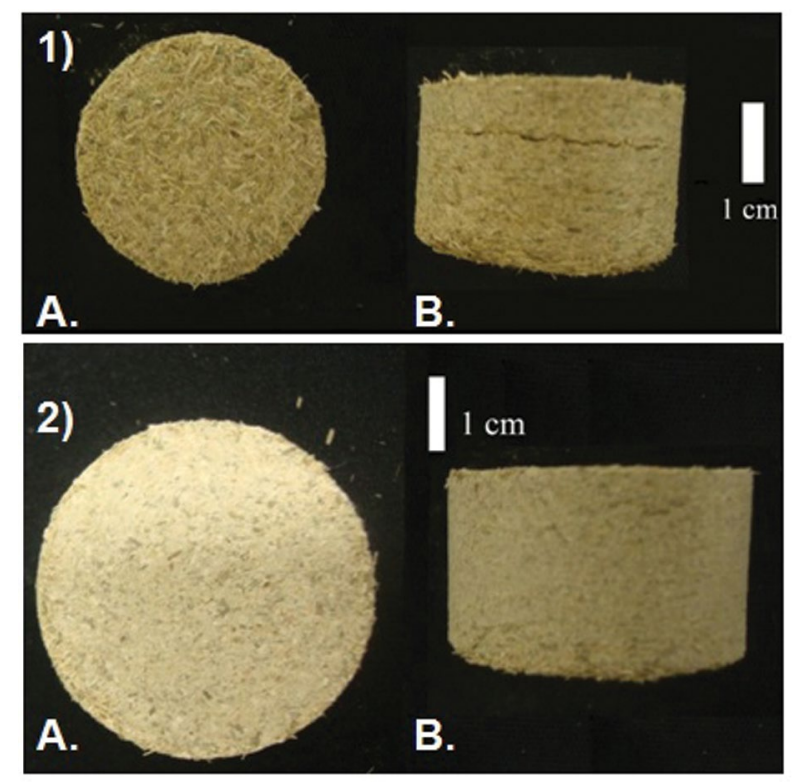

Figura 1. 1) Briquetes de Bambusa vulgaris var. vitatta e 2) Briquetes de Bambusa vulgaris var. vulgaris em dois planos, A. transversal e B. longitudinal.

Figure 1. 1) Bambusa vulgaris var. vitatta briquettes and 2) Bambusa vulgaris var. vulgaris briquettes in two planes, A. transversal and B. longitudinal. 
AMARAL, P.M. et al. Caracterização de briquetes de duas variedades de bambu

Tabela 4. Expansão diametral e longitudinal dos briquetes pós-compactação após 96 horas.

Table 4. Briquettes expansion 96 hours after the compaction.

\begin{tabular}{ccc}
\hline Espécie & Expansão diametral após 96 horas (\%) & Expansão longitudinal após 96 horas (\%) \\
\hline Variedade vitatta & $0,22 \pm 0,03$ & $7,30 \pm 0,60$ \\
Variedade vulgaris & $0,47 \pm 0,06$ & $7,55 \pm 0,57$ \\
\hline
\end{tabular}

Segundo Buzin (2009), considera-se normal uma expansão de até $20 \%$, mostrando, então, que os dois materiais aqui estudados sofreram expansões que se encontram dentro dos valores considerados normais. Essa propriedade deve ser cuidadosamente observada, pois seus valores afetam diretamente na resistência mecânica dos briquetes (Chrisostomo, 2011). Sabe-se também que quanto menor a expansão volumétrica dos briquetes mais vantajosa é a sua produção, uma vez que os compactos não sofrerão alterações em seu aspecto e ocuparão menor espaço em seu transporte.

\subsection{Ensaio Mecânico}

Na Figura 2, tem-se os resultados do ensaio mecânico da variedade vitatta em relação à força máxima média suportada e a tensão máxima média.
Em relação à variedade vulgaris, os resultados encontram-se no gráfico (Figura 3 ).

Nos ensaios mecânicos, o material da variedade vitatta mostrou-se mais resistente à força máxima média que o material da variedade vulgaris, sendo os valors de 510,44 e $499,80 \mathrm{~N}$, respectivamente. Essa diferença nas forças máximas médias suportadas, ao contrário do que se esperava, não pode ser explicada pela maior interação entre as partículas e sua maior proximidade, o que seria resultado da menor granulometria do material. Uma possível explicação pode ser exatamente a diferente variedade da espécie em questão. Acredita-se, então, que há uma maior quantidade de feixes vasculares na var. vitatta, o que pode garantir maior resistência mecânica externa (Carlessi, 2010).

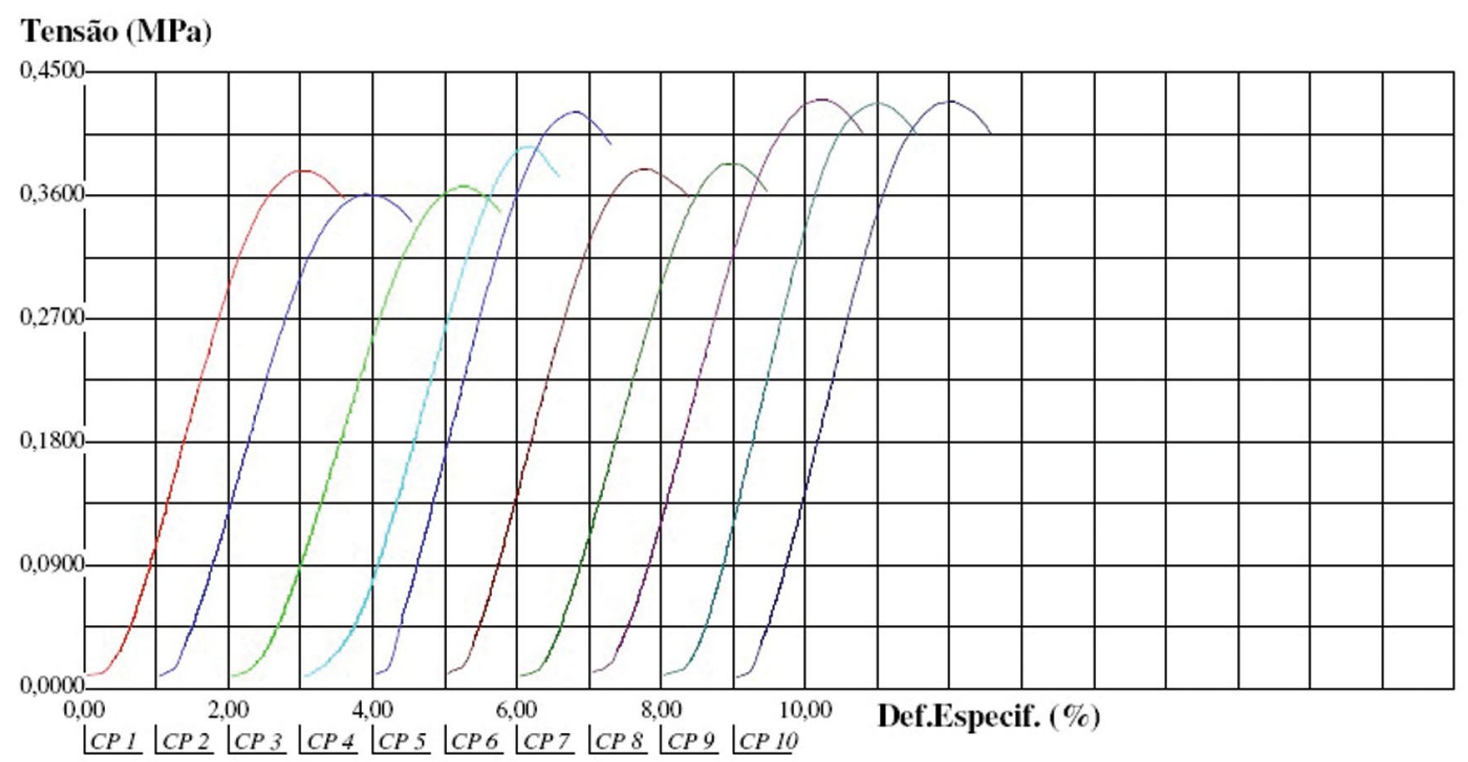

Figura 2. Gráfico de tensão x deformação específica dos briquetes Bambusa vulgaris var. vitatta.

Figure 2. Strain vs. stress for briquettes of Bambusa vulgaris var. vitatta. 


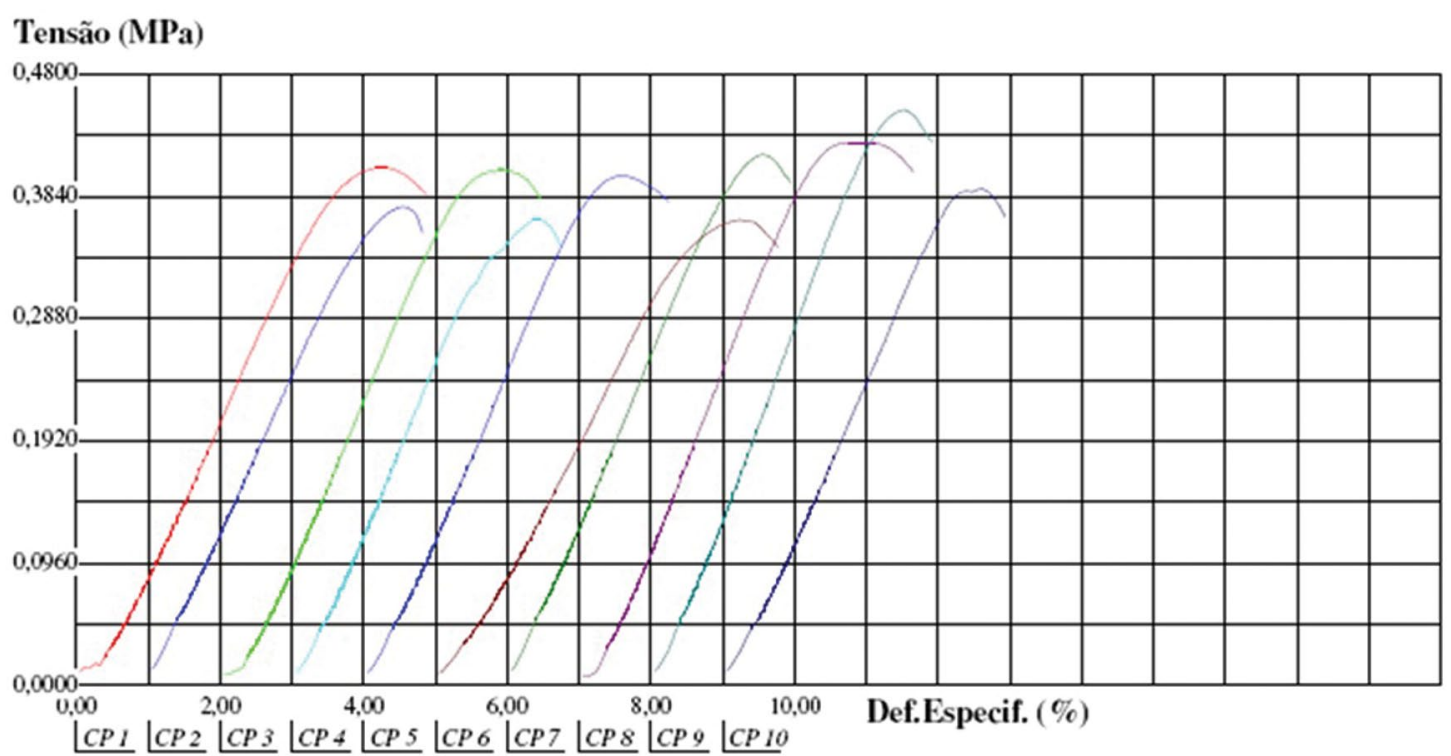

Figura 3. Gráfico de tensão x deformação específica dos briquetes Bambusa vulgaris var. vulgaris.

Figure 3. Strain vs. stress for briquettes of Bambusa vulgaris var. vulgaris.

Gonçalves et al. (2013) encontraram para briquetes de serragem de Eucalyptus grandis um valor de $649,10 \mathrm{~N}$, o qual se aproxima do valor médio encontrado neste trabalho para os briquetes produzidos a partir das duas variedades de bambu.

A avaliação mecânica dos briquetes tem sua importância como ferramenta para o direcionamento do uso final deste material compacto, podendo ser este doméstico ou industrial (Protásio et al., 2014).

\section{CONCLUSÃO}

Foi possível a formação de briquetes das duas variedades de bambu aqui estudadas, sendo elas Bambusa vulgaris var. vitatta e Bambusa vulgaris var. vulgaris.

As variedades de espécie de bambu podem influenciar no comportamento químico, físico e mecânico dos briquetes produzidos.

As duas espécies estudadas, Bambusa vulgaris var. vitatta e Bambusa vulgaris var. vulgaris, apresentaram predisposição a uso como combustível sólido em relação às propriedades aqui estudadas, constituindo assim uma alternativa viável em relação à produção de energia à partir de matérias-primas não renováveis.

\section{REFERÊNCIAS BIBLIOGRÁFICAS}

ALBERTINI, J. L. A. Cultura do bambu. Informe de Pesquisa, v. 3, n. 22, p. 1-7, 1979.

ASSOCIAÇÃO BRASILEIRA DE NORMAS TÉCNICAS - ABNT. NBR 6922 - Carvão vegetal: determinação da densidade a granel. Rio de Janeiro, 1981.

NBR 8112 - Carvão vegetal: análise imediata. Rio de Janeiro, 1986.

NBR 7217 - Agregados: determinação da composição granulométrica. Rio de Janeiro, 1987.

AZZINI, A.; CIARAMELLO, D.; NAGAI, V. Densidade básica e dimensões de bambu do gênero Guadua. Bragantia, v. 36, p. 1-5, 1977.

BARROS, J.L. Caracterização de blendas e briquetes de carvões vegetal e mineral. 2014. 121 f. Dissertação (Mestrado em Ciências dos Materiais) - Centro de Ciências e Tecnologias para Sustentabilidade, Universidade Federal de São Carlos, Sorocaba.

BIANCHINI, A. et al. A influência dos extrativos do bagaço de cana-de-açúcar na produção de briquetes. In: ENCONTRO BRASILEIRO EM MADEIRAS E ESTRUTURAS DE MADEIRA EBRAMEM, 13., 2012, Vitória. Anais... Vitória: UFES, 2012. v. 1, p. 1-8. 
BUZIN, P.J.W.K. Desenvolvimento de briquetes autorredutores a partir de carepas de processamento siderúrgico para utilização em forno elétrico a arco. 2009. $138 \mathrm{f}$. Tese (Mestrado em Engenharia de Minas) - Centro de Tecnologia da Escola de Engenharia, Universidade do Rio Grande do Sul, Porto Alegre.

CÂNDIDO, J.F.; RIBEIRO, J.B. Culturas florestais I (algaroba - aroeira - bambu). Viçosa-MG: UFV, 1991. $44 \mathrm{p}$.

CARLESSI, A.S. Avaliação da substituição de fibra de aço por fibra de bambu em piso de concreto não armado. 2010. 67 f. Trabalho de conclusão de curso (Obtenção do grau de Engenheiro Civil) Universidade do Extremo Sul Catarinense, Criciúma.

CARVALHO, E.A.; BRINCK, V. Briquetagem. Rio de Janeiro: Centro de Tecnologia Mineral, 2004. p. 613-617.

CERRI, C. (Bambu) o rei do oriente, SP, Brasil. Revista Globo Rural, v. 6, n. 65, p. 26-31, 1991.

CHRISOSTOMO, W. Estudo da compactação de resíduos lignocelulósicos para utilização como combustível sólido. 2011. 80 f. Dissertação (Mestrado em Ciência dos Materiais) - Universidade Federal de São Carlos, Sorocaba.

CIARAMELLO, D. Bambu tem mil e uma possibilidades. Cooper-cotia, p. 24-5, março, 1968.

CORTEZ, C.L. et al. Análise do processo do eucalipto no sistema "short rotation" para uso como combustível em uma usina termoelétrica. In: CONGRESSO INTERNACIONAL DE BIOENERGIA, 4., 2009. Anais... Curitiba: UFPR: FUPEF, 2009. p. 1-7.

COSTA, T.M.S. Estudo da viabilidade técnica do emprego do bambu da espécie Bambusa vulgaris Schard. como carvão vegetal. 2004. 74 f. Dissertação (Mestrado em Ciência na área de Tecnologia Nuclear) - Instituto de Pesquisas Energéticas e Nucleares, Universidade de São Paulo, São Paulo.

FLORES, W.P. et al. Caracterização de briquetes de Eucalyptus sp. e Pinus sp. Revista da Madeira, não paginado, ago. 2010.
GOMIDE, J.L.; OLIVEIRA, R.C.; COLODETTE, J.L. Influência da idade de Bambusa vulgaris nas suas características químicas e anatômicas, visando a produção de polpa celulósica. In: CONGRESSO ANUAL DA ABCP, 14., 1981, São Paulo. Anais... São Paulo: ABCP, 1981. v. 1, p. 5-29.

GONÇALVES, B.F. et al. Caracterização e comparação entre diferentes granulometrias de serragem de Eucalyptus grandis para confecção de briquetes. Revista do Instituto Florestal, v. 25, n. 2, p. 205-213, 2013.

JUNIOR, M.G. et al. Caracterização físico-química de fibras e polpas de Bambusa vulgaris Schrad para utilização em compósitos poliméricos. Revista LatinAm. Metal. Mat., v. 33, n. 1, p. 33-42, 2011.

KLEINLEIN, W.A. Caracterização energética de biomassas amazônicas. 2010. 63 f. Dissertação (Mestrado em Engenharia Mecânica) - Instituto de Tecnologia, Universidade Federal do Pará, Belém.

LIU, Z. et al. Combustion characteristics of bamboo-biochars. Bioresource Technology, v. 167, p. 94-99, 2014.

PINHEIRO, G.F. et al. Densidade energética de resíduos vegetais. Biomassa \& Energia, v. 2, n. 2, p. 113-123, 2005.

PROTÁSIO, T.P. et al. Efeitos da torrefação nas propriedades físicas, químicas e energéticas de briquetes de serragem de Eucalyptus sp. In: CONGRESSO INTERNACIONAL DE BIOENERGIA, 6., 2011, Curitiba. Anais... Curitiba, 2011.

. et al. Efeito da idade e clone na qualidade da madeira de Eucalyptus spp. visando à produção de bioenergia. Ciência Florestal, v. 24, n. 2, p. 465-477, 2014.

QUIRINO, W.F.; BRITO, J.O. Características e índice de combustão de briquetes de carvão vegetal. Brasília, DF: Instituto Brasileiro de Meio Ambiente e Recursos Naturais Renováveis, 1991. $19 \mathrm{p}$. 